\title{
Mechanical CPR: Who? When? How?
}

\author{
Kurtis Poole ${ }^{1,2}$, Keith Couper ${ }^{1,3}$, Michael A. Smyth ${ }^{1,4}$, Joyce Yeung ${ }^{1,3}$ and Gavin D. Perkins ${ }^{1,3^{*}}$
}

\begin{abstract}
In cardiac arrest, high quality cardiopulmonary resuscitation (CPR) is a key determinant of patient survival. However, delivery of effective chest compressions is often inconsistent, subject to fatigue and practically challenging. Mechanical CPR devices provide an automated way to deliver high-quality CPR. However, large randomised controlled trials of the routine use of mechanical devices in the out-of-hospital setting have found no evidence of improved patient outcome in patients treated with mechanical CPR, compared with manual CPR. The limited data on use during in-hospital cardiac arrest provides preliminary data supporting use of mechanical devices, but this needs to be robustly tested in randomised controlled trials.

In situations where high-quality manual chest compressions cannot be safely delivered, the use of a mechanical device may be a reasonable clinical approach. Examples of such situations include ambulance transportation, primary percutaneous coronary intervention, as a bridge to extracorporeal CPR and to facilitate uncontrolled organ donation after circulatory death.

The precise time point during a cardiac arrest at which to deploy a mechanical device is uncertain, particularly in patients presenting in a shockable rhythm. The deployment process requires interruptions in chest compression, which may be harmful if the pause is prolonged. It is recommended that use of mechanical devices should occur only in systems where quality assurance mechanisms are in place to monitor and manage pauses associated with deployment.

In summary, mechanical CPR devices may provide a useful adjunct to standard treatment in specific situations, but current evidence does not support their routine use.
\end{abstract}

Keywords: Cardiac arrest, Cardiopulmonary resuscitation, Mechanical CPR, Review

\section{Background}

High-quality chest compressions are a critical component in the cardiac arrest chain of survival [1]. Despite its importance, the sustained delivery of high-quality cardiopulmonary resuscitation (CPR) is infrequently achieved in clinical practice $[2,3]$.

Mechanical chest compression devices deliver high-quality external chest compressions, in place of a human rescuer. A number of devices are currently marketed, but devices can be broadly categorised as load distributing band or piston devices, based on the mechanism that is used to deliver compressions. The Autopulse (Zoll Medical, Chelmsford, MA, USA) is a load distributing band device, which consists of a large backplate that is positioned behind the patient and a band that encircles the patient's chest to deliver compressions at a rate of 80

\footnotetext{
* Correspondence: g.d.perkins@warwick.ac.uk

${ }^{1}$ Warwick Medical School, University of Warwick, Coventry, UK

${ }^{3}$ University Hospitals Birmingham NHS Foundation Trust, Birmingham, UK

Full list of author information is available at the end of the article
}

per minute and a depth of $20 \%$ of the anterior-posterior chest height. The LUCAS (Physio-Control Inc./Jolife AB, Lund, Sweden) is an example of a piston device, which also incorporates a mechanism for active chest recoil. It consists of two parts (a backplate and the piston mechanism), which link together to encircle the patient. The device consistently delivers compressions at a rate of 102 per minute and a depth of $5.3 \mathrm{~cm}$ in patients with a sternal height greater than $18.5 \mathrm{~cm}$. The key theoretical benefit to the use of such devices is their ability to consistently deliver high-quality chest compressions, which has been associated with improved intra-arrest haemodynamic profiles $[4,5]$.

The purpose of this review is to provide an update on mechanical device use for both out-of-hospital cardiac arrest (OHCA) and in-hospital cardiac arrest (IHCA), an overview on device use in special circumstances, and guidance on deployment in the clinical setting.

(C) The Author(s). 2018 Open Access This article is distributed under the terms of the Creative Commons Attribution 4.0 International License (http://creativecommons.org/licenses/by/4.0/), which permits unrestricted use, distribution, and reproduction in any medium, provided you give appropriate credit to the original author(s) and the source, provide a link to the Creative Commons license, and indicate if changes were made. The Creative Commons Public Domain Dedication waiver (http://creativecommons.org/publicdomain/zero/1.0/) applies to the data made available in this article, unless otherwise stated. 


\section{The importance of high quality CPR}

International guidelines highlight the importance of high-quality chest compressions, which are defined as compressions at a depth of 5-6 cm and a rate of 100120 per minute, allowing full chest recoil between compressions, and minimisation of interruptions [6, 7].

Despite consistent observational data showing the association between CPR quality and patient outcome [8, 9], the delivery of high-quality manual chest compressions is challenging in both the out-of-hospital and in-hospital settings [2, 3]. Specific barriers include provider fatigue $[10,11]$, physical effort to overcome stiffness of the patient's thoracic cage [12], and compressible underlying surfaces, such as mattresses, which can lead to shallow chest compressions $[13,14]$. For example, in an analysis of 9136 OHCA patients, only 45\% received the recommended guideline chest compression depth [15].

In contrast to manual chest compressions, mechanical devices are not subject to the physical limitations of the rescuer and are able to consistently deliver high-quality chest compressions.

\section{Current treatment recommendations}

In 2015, the International Liaison Committee on Resuscitation's (ILCOR) consensus on science and treatment recommendation process evaluated the use of mechanical chest compression devices in clinical practice [16]. The evidence evaluation process made a single treatment recommendation to cover all settings and all mechanical device types.

Based on the expert review of the available data, ILCOR made a weak recommendation (moderate quality evidence) against the routine use of mechanical devices in clinical practice. However, the review acknowledged situations where the delivery of high quality manual chest compressions may be impractical or dangerous to rescuers. In such circumstances, ILCOR made a weak recommendation based on low-quality evidence supporting the use of mechanical devices. The commentary accompanying the treatment recommendation highlighted concerns that the deployment of mechanical devices without appropriate training might cause patient harm through an increase in no-flow time during the early part of the cardiac arrest and delay defibrillation in patients with a shockable rhythm.

\section{Out-of-hospital cardiac arrest \\ Routine deployment in OHCA}

The routine deployment of mechanical devices has been robustly tested in the pre-hospital setting in large high-quality randomised controlled trials. In 2014-2015, the CIRC (Circulation Improving Resuscitation Care) [17], LINC (LUCAS in Cardiac Arrest) [18], and PARAMEDIC (Prehospital Randomised Assessment of a Mechanical
Chest Compression Device in Cardiac Arrest) [19] trials were published. These studies, alongside two earlier small randomised studies [20, 21], are summarised in Table 1.

The CIRC trial was an industry sponsored trial, designed to determine equivalence, superiority, or inferiority in survival to hospital discharge for OHCA patients who were randomised in a 1:1 ratio to receive either manual CPR or Autopulse CPR [17]. The study was terminated early, in accordance with pre-defined stopping rules, after 4753 randomised patients were enrolled. Of those randomised, 4231 were included in the intention-to-treat analysis. Overall, manual CPR showed a numeric increase in survival to hospital discharge compared to Autopulse CPR (11.0 versus 9.4\%). The adjusted odds ratio (OR) was 1.06 (95\% confidence interval (CI) $0.83,1.37$ ) after adjustment for covariates and interim analyses. This fell within the pre-defined equivalence region (OR 0.69-1.44), although the width of the equivalence margin incorporates the potential for both significant harm and benefit [22]. Overall hospital survival rate was higher than that reported in similar studies (PARAMEDIC 30 day survival 6.6\%; LINC hospital survival $8.0 \%$ ). This may reflect the stringent study inclusion criteria and intensive training and oversight by the study team which emphasised the importance of high-quality CPR.

The LINC trial was also an industry-sponsored efficacy trial, in which OHCA patients were randomised in a 1:1 ratio to receive either LUCAS or manual CPR [18]. The trial used a modified treatment algorithm for the LUCAS arm that incorporated defibrillation without rhythm assessment and 3-minute periods between rhythm assessments. The study randomised 2593 patients, of which 1589 were included in the intention-to-treat analysis. In relation to the primary outcome of 4-h survival, LUCAS was not superior to manual chest compressions (treatment difference $0.05 \%, 95 \% \mathrm{CI}-3.3,3.2$ ).

The PARAMEDIC study was an academic pragmatic cluster randomised trial, in which ambulance vehicles were randomised in a 2:1 ratio to receive either manual CPR or LUCAS CPR. The treatment allocation of the individual patient was determined by the first vehicle to arrive on scene. The study included 4471 patients, of which 4470 were included in the primary analysis. In relation to the primary outcome of 30-day survival, LUCAS was not superior to manual compressions (adjusted OR $0.86,95 \%$ CI $0.64,1.15)$. The study experienced a high incidence of non-compliance in the LUCAS CPR arm, such that only $60 \%$ received mechanical CPR. A CACE (compiler average causal effect) analysis, which accounts for non-compliance, generated similar findings to the main analysis [23].

The PARAMEDIC study also collected cost-effectiveness and quality of life data following hospital discharge [24-26]. The long-term (up to 12 months) outcome analysis found 
Table 1 Summary of randomized controlled trials comparing routine use of mechanical CPR with manual CPR in OHCA

\begin{tabular}{|c|c|c|c|c|c|}
\hline & \multicolumn{2}{|c|}{ Load-distributing band trials } & \multicolumn{3}{|l|}{ Piston-device trials } \\
\hline & Hallstrom et al. [20] ${ }^{a}$ & CIRC [17] & Smekal et al. [21] & LINC [18] & PARAMEDIC [19] \\
\hline Design & $\begin{array}{l}\text { Efficacy superiortity } \\
\text { multicentre RCT }\end{array}$ & $\begin{array}{l}\text { Efficacy equivalence } \\
\text { multicentre RCT }\end{array}$ & Pilot multicentre RCT & $\begin{array}{l}\text { Efficacy superiority } \\
\text { multicentre RCT }\end{array}$ & $\begin{array}{l}\text { Effectiveness superiority } \\
\text { multicentre RCT }\end{array}$ \\
\hline Randomisation & $\begin{array}{l}\text { Cluster- EMS station(s) } \\
\text { (ratio 1:1) }\end{array}$ & Patient (ratio 1:1) & Patient (ratio 1:1) & Patient (ratio 1:1) & $\begin{array}{l}\text { Cluster- ambulance (ratio } 2 \\
\text { manual:1 mechanical) }\end{array}$ \\
\hline Inclusion criteria & $\begin{array}{l}\text { Adult non-traumatic } \\
\text { OHCA }\end{array}$ & $\begin{array}{l}\text { Adult OHCA of cardiac } \\
\text { aetiology. EMS arrival } \\
\text { time } \leq 16 \mathrm{~min}\end{array}$ & $\begin{array}{l}\text { Adult non-traumatic } \\
\text { OHCA }\end{array}$ & $\begin{array}{l}\text { Adult unexpected } \\
\text { non-traumatic OHCA } \\
\text { where resuscitation } \\
\text { was appropriate }\end{array}$ & $\begin{array}{l}\text { Adult non-traumatic } \\
\text { OHCA where a trial } \\
\text { vehicle was first } \\
\text { ambulance on scene }\end{array}$ \\
\hline $\begin{array}{l}\text { Number of cases } \\
\text { analysed }\end{array}$ & $\begin{array}{l}1071 \text { (1071 } \\
\text { randomised) }\end{array}$ & 4231 (4753 randomised) & 149 & 2589 (2593 randomised) & 4470 (4471 randomised) \\
\hline Setting & US/Canada & US /Europe & Sweden & Europe & UK \\
\hline Sponsor & Industry & Industry & Academic $^{b}$ & Industry & Academic \\
\hline Device used & Autopulse & Autopulse & LUCAS & LUCAS & LUCAS \\
\hline Primary outcome & $\begin{array}{l}\text { 4-h survival: } \\
\text { Manual } 29.5 \% \text { vs } \\
\text { mechanical } 28.5 \% \text {, } \\
p=0.74\end{array}$ & $\begin{array}{l}\text { STD } \\
\text { Manual } 11.0 \% \text { vs } \\
\text { mechanical } 9.4 \% \text {, adj. } \\
\text { OR } 1.06(95 \% \mathrm{Cl} 0.83 \text {, } \\
1.37)^{\mathrm{C}}\end{array}$ & Not specified & $\begin{array}{l}\text { 4-h survival } \\
\text { Manual } 23.7 \% \text { vs } \\
\text { mechanical } 23.6 \% \text {, } \\
\text { treatment difference } \\
-0.05(95 \% \mathrm{Cl}-3.3,3.2)\end{array}$ & $\begin{array}{l}\text { 30-day survival } \\
\text { Manual } 7 \% \text { vs mechanical } \\
6 \% \text {, adj. OR } 0.86(95 \% \mathrm{Cl} \\
0.64,1.15)\end{array}$ \\
\hline $\begin{array}{l}\text { Key secondary } \\
\text { outcomes }\end{array}$ & $\begin{array}{l}\text { Cardiac aetiology } \\
\text { group ( } n=767) \text { : } \\
\text { STD: Manual 9.9\% } \\
\text { vs mechanical } 5.8 \%, \\
p=0.06 \text { (adjusted) } \\
\text { Good neurological } \\
\text { outcome: Manual } \\
7.5 \% \text { vs mechanical } \\
3.1 \%, p=0.006\end{array}$ & $\begin{array}{l}\text { Sustained ROSC: Manual } \\
32.3 \% \text { vs mechanical } \\
28.6 \% \text {, adj. OR } 0.84 \\
\text { ( } 95 \% \text { Cl } 0.73,0.96) \\
\text { Good neurological } \\
\text { outcome in survivors: } \\
\text { Manual } 48.1 \% \text { vs } \\
\text { mechanical } 44.4 \% \text {, } \\
\text { adj. OR } 0.80(95 \% \\
\text { Cl } 0.47,1.37)\end{array}$ & $\begin{array}{l}\text { ROSC: Manual } 32 \% \\
\text { vs mechanical } 41 \% \text {, } \\
p=0.30 \\
\text { STD: Manual } 10 \% \\
\text { vs mechanical } 8 \%, \\
p=0.78\end{array}$ & $\begin{array}{l}\text { STD: Manual } 9.2 \% \text { vs } \\
\text { mechanical 9.0\%, } \\
\text { treatment difference } \\
-0.15 \text { ( } 95 \% \mathrm{Cl}-2.4,2.1) \\
\text { Good neurological } \\
\text { outcome: Manual } 7.3 \% \\
\text { vs mechanical } 8.1 \% \text {, } \\
\text { treatment difference } \\
0.78 \text { ( } 95 \% \mathrm{Cl}-1.3,2.8)\end{array}$ & $\begin{array}{l}\text { ROSC: Manual } 31 \% \text { vs } \\
\text { mechanical 32\%, adj. OR } \\
0.99 \text { ( } 95 \% \text { Cl 0.86, 1.14) } \\
\text { Good neurological outcome: } \\
\text { Manual } 6 \% \text { vs mechanical } \\
\text { 5\%, adj. OR } 0.72 \text { ( } 95 \% \text { Cl 0.52, } \\
\text { 0.99) }\end{array}$ \\
\hline
\end{tabular}

${ }^{\text {a } T r i a l ~ s t o p p e d ~ e a r l y ~ b y ~ d a t a ~ m o n i t o r i n g ~ b o a r d ~}$

bone author received consulting fee from device manufacturer

'Primary outcome result within pre-specified boundary of equivalence. Trial stopped early in accordance with pre-specified stopping rule

EMS Emerency Medical Service, OHCA out-of-hospital cardiac arrest, RCT randomised controlled trial, STD survival to discharge, adj OR adjusted odds ratio

no clinically important differences between groups in relation to outcomes such as survival, neurological outcome, and quality of life at 3 months and 12 months, although the analysis was subject to a high risk of attrition bias [24]. The cost-effectiveness analysis found that routine use of mechanical CPR devices in the out-of-hospital setting was not cost-effective [25].

A systematic review and meta-analysis by Gates et al. [22] incorporated all five randomised controlled trials, as summarised in Table 1, with a total patient population of 12,206. The random-effects meta-analysis found that mechanical CPR was not superior to manual CPR, in relation to key outcomes such as return of spontaneous circulation (OR $0.96,95 \%$ CI $0.85,1.10$ ), survival at hospital discharge/30 days (OR $0.89,95 \%$ CI $0.77,1.02$ ), or good neurological outcome (OR 0.76, 95\% CI 0.53, 1.11).

In summary, these data do not support the routine use of mechanical CPR in OHCA.

\section{Use during transfer to hospital}

The Universal Termination of Resuscitation rule guides clinical teams when to consider transport from scene of the cardiac arrest to hospital with on-going CPR [27]. Other indications for transport include situations where potentially lifesaving treatments cannot be delivered outside a hospital, such as extra-corporeal CPR, re-warming after hypothermic cardiac arrest, and invasive procedures (e.g. primary percutaneous coronary intervention (pPCI)) [28]. The process of intra-arrest transport typically requires two phases: extrication of the patient to the ambulance and vehicular transfer to the hospital. In each of these phases, a key challenge for the EMS crew is the safe ongoing delivery of high-quality CPR.

The majority of OHCAs occur in the patient's home [29]. As such, a key challenge in the extrication stage is manoeuvring past obstacles and downstairs whilst continuing to deliver CPR. In an observational study, researchers analysed the pauses associated with this process prior to and following the introduction of mechanical CPR to facilitate the extrication process [30]. In the first period where manual CPR was provided, the median chest compression pause during extrication was 270 s (interquartile range (IQR) 201, 387), with some pauses recorded as being in excess of $10 \mathrm{~min}$. In 
contrast, after introduction of mechanical CPR, chest compressions were delivered continuously during extrication, except for the pause required to deploy the mechanical device (median 39 s (IQR 29, 47).

For the vehicular transfer to hospital stage, there are three main concerns. Firstly, delivery of manual CPR in a moving vehicle is inherently unsafe, and exposes both the patient and EMS provider to risk of injury or death $[31,32]$. Secondly, there is a risk of suboptimal CPR delivery due to acceleration forces during ambulance transport [33]. However, evidence from clinical studies has been mixed with some reporting a similar quality of manual chest compressions prior to and during transfer, whilst other studies have reported either a transfer related deterioration or increased variability in quality [30, 34-36]. Finally space consideration, such as in the context of transport by helicopter, may render delivery of manual CPR difficult or impossible. In this setting, mechanical chest compression devices have been used to effectively deliver ongoing CPR during transport [37].

Based on these data, in particular the safety concerns associated with the delivery of manual CPR during transport, it would seem reasonable to consider the use of mechanical CPR during transport to hospital.

\section{Use in the emergency department}

The emergency department (ED) cardiac arrest population comprises both OHCA patients that did have a pre-hospital ROSC and patients that arrest in the emergency department. In view of limited personnel and a case-mix that likely includes prolonged cardiac arrests, mechanical device use in the ED may appear an attractive solution. A recent multi-centre Japanese observational study analysed the outcome of 6537 cardiac arrest patients (5619 manual CPR, 918 mechanical CPR) treated in the ED [38]. The use of a device was associated with reduced likelihood of ROSC (unadjusted OR 0.90, 95\% CI 0.77, 1.06; adjusted 0.71, 95\% CI 0.53, 0.94) and hospital survival (unadjusted OR 0.97, 95\% CI 0.62, 1.51 ; adjusted $0.40,95 \%$ CI $0.20,0.78$ ). However, the decision as to whether or not to use a mechanical device was made on a patient by patient basis, such that there is a high risk that selective enrolment introduced unmeasured confounding variables which may have biased the results.

In a before-after study in two Singaporean hospitals, researchers compared patient outcomes prior to and following the implementation of the Autopulse device as part of the treatment for ED cardiac arrests [39]. In total, 1011 (459 manual CPR period; 552 mechanical CPR period) patients were studied. Unadjusted ORs show an association between treatment in the mechanical CPR period and improved ROSC (OR 1.89, 95\% CI 1.43, 2.50), hospital survival (OR 2.55, 95\% CI 1.00, 6.47), and good neurological outcome (OR 8.7, 95\% CI 1.1, 71.6), but the interpretation of these findings is complicated by marked differences in baseline patient characteristics (e.g. initial rhythm, arrest location). Adjusted analyses showed an association between treatment in the mechanical CPR period and ROSC (OR 1.60, 95\% 1.16, 2.22), but no association was observed in relation to any other outcome.

The reason for the apparent contrast in findings may reflect differences in patient population, study risk of bias (selection bias, effect of unmeasured confounders), or the strategy used to deploy the mechanical device. In particular, the team that deployed the device in Ong et al.'s study [40] had received focussed team training to optimise device deployment, thereby minimising pauses associated with its use. Overall, the findings of these studies with their inherent risk of bias do not support the routine use of mechanical CPR in the ED.

\section{In-hospital cardiac arrest}

In contrast to the OHCA setting, few studies have sought to evaluate the routine use of mechanical CPR in the IHCA setting. A recent systematic review and meta-analysis identified only three randomised controlled trials which enrolled 234 patients [41].

The largest of these trials, and the only study published in the last 20 years, enrolled 150 in-hospital cardiac arrest patients who were randomised to receive either mechanical CPR delivered by a piston device or manual CPR [42]. The study report is available only in Chinese. After translation, unfortunately, key patient characteristics, such as initial rhythm, are not reported. The study reported that the use of a mechanical device improved survival to hospital discharge (OR 2.81, 95\% CI 1.26, 6.24). This trial, alongside the two other trials [43, 44], are summarised in Table 2.

The meta-analysis of the three randomised trials alongside six (455 patients) observational studies found very low quality evidence supporting an association between mechanical CPR use and increased likelihood of ROSC (OR 2.14, 95\% CI 1.11, 4.13) and survival to hospital discharge/30 days (OR 2.34, 95\% CI 1.42, 3.85) [41]. Neurological outcome was not assessed in any study. The results of the meta-analysis were broadly consistent between the sub-groups of randomised controlled trials and observational studies.

These findings seemingly contrast with research findings from out-of-hospital studies [22]. Reasons for this apparent discrepancy may reflect differences in either the quality of evidence or clinical characteristics between the two settings, such that mechanical devices may be more effective than manual chest compressions in the hospital setting. Examples of such characteristics include the opportunity for early device deployment and the challenges of delivering effective manual chest compressions on a bed mattress. 
Table 2 Summary of randomized controlled trials comparing routine use of mechanical CPR with manual CPR in IHCA

\begin{tabular}{|c|c|c|c|}
\hline & \multirow{2}{*}{$\begin{array}{l}\text { Load-distributing band trials } \\
\text { Halperin et al. [44] }\end{array}$} & \multicolumn{2}{|l|}{ Piston-device trials } \\
\hline & & Taylor et al. [43] & Lu et al. [42] ${ }^{a}$ \\
\hline Design & Efficacy superiortity single-centre RCT & Efficacy superiortity single-centre RCT & Efficacy superiority single-centre RCT \\
\hline Randomisation & Patient (ratio 1:1) & Patient (ratio 1:1) & Patient (ratio 1:1) \\
\hline Inclusion criteria & $\begin{array}{l}\text { IHCA of less than 20-min duration } \\
\text { following tracheal intubation and } \\
\text { adrenaline administration }\end{array}$ & IHCA of less than 10-min duration & IHCA of less than 10-min duration \\
\hline Number of cases analysed & 34 & 50 & 150 \\
\hline Setting & US & US & China \\
\hline Sponsor & Academic $^{b}$ & Academic & Unclear \\
\hline Device used & Load-distributing band device & Thumper device & Thumper device \\
\hline Primary outcome & $\begin{array}{l}\text { ROSC } \\
\text { Manual } 18 \% \text { vs mechanical } 47 \% \text {, } \\
\text { OR } 4.15(95 \% \text { Cl } 0.86,19.92)\end{array}$ & $\begin{array}{l}\text { One-hour survival } \\
\text { Manual 38\% vs mechanical 42\%, } \\
\text { OR } 1.14(95 \% \text { Cl } 0.37,3.55)\end{array}$ & $\begin{array}{l}\text { STD } \\
\text { Manual 15\% vs mechanical 33\%, } \\
\text { OR } 2.81(95 \% \text { Cl } 1.26,6.24)\end{array}$ \\
\hline Key secondary outcomes & $\begin{array}{l}\text { 24-h survival: Manual } 6 \% \text { vs } \\
\text { mechanical } 18 \%\end{array}$ & $\begin{array}{l}\text { STD: Manual 8\% vs mechanical } \\
13 \% \text {, OR } 1.71(95 \% \mathrm{Cl} 0.26,11.26)\end{array}$ & $\begin{array}{l}\text { ROSC: Manual 38\% vs mechanical } \\
55 \% \text {, OR } 2.03(95 \% \mathrm{Cl} 1.06,3.90)\end{array}$ \\
\hline
\end{tabular}

apublished only in Chinese

${ }^{b}$ Nine authors report equity interest in company holding device patent

IHCA in-hospital cardiac arrest, RCT randomised controlled trial, STD survival to discharge

Based on this discrepancy, the need for a randomised controlled trial in the in-hospital setting was recently highlighted as a research priority [45]. The ongoing COMPRESS-RCT (ISRCTN38139840) study is assessing the feasibility of undertaking such a trial.

\section{Risk of injury during mechanical device use}

Injuries secondary to manual chest compression are common and well-reported [46]. Common injuries include fractures (rib, sternal), pneumothoraces, and visceral organ damage (liver, spleen, heart) [46-48]. Several case reports have purportedly linked mechanical device use with clinically important injuries, thereby driving concern that mechanical devices may increase risk of injury compared with manual CPR [49-51]. Whilst evidence from cohort studies has produced mixed results, interpretation of these studies is challenging as they are prone to selection bias and the quality of manual CPR delivered, as the comparator group, is generally not recorded [52-55]. The PARAMEDIC, LINC, and CIRC trials were designed to examine the clinical effectiveness of mechanical devices, rather than to specifically examine injury, but it is noteworthy that these trials did not report a difference in injury patterns or severity between patients receiving manual and mechanical chest compression [17-19].

Koster et al. recently published a non-inferiority randomised controlled trial that provided the most robust evidence in relation to injury attributable to mechanical chest compression devices [56]. In total, 374 patients were randomised to receive LUCAS CPR, Autopulse CPR, or to continue to receive manual CPR [56]. The primary outcome was serious or life-threatening resuscitation-related visceral organ damage. Outcome data were available for $90 \%$ of participants. Compared to manual CPR, the non-inferiority analysis showed that LUCAS did not increase the risk of injury. However, an increase in injury could not be ruled out with the Autopulse device. The depth of manual chest compressions delivered in the manual CPR arm was $48 \mathrm{~mm}$ (SD 9), which is slightly lower than the current recommended target depth of $50 \mathrm{~mm}$ [6].

\section{Adjunct to advanced treatments pPCI and CT scan}

Delivery of high quality manual chest compressions during imaging procedures, such as coronary angiography or CT scan, is practically challenging due to the required positioning of the radiology equipment. Several case series describe the experience of specialist centres in performing intra-arrest coronary angiography and $\mathrm{pPCI}$ facilitated by mechanical CPR, with reported hospital survival rates of approximately 25\% [57-59]. Wagner et al. [58] acknowledge that movement during CPR increases the complexity of the procedure, but recommend strategies such as a brief CPR pause during stenting to overcome this challenge. Whether the routine transfer of patients in refractory cardiac arrest for pPCI during on-going CPR improves patient outcome remains to be determined.

Transporting a patient in cardiac arrest to the CT scanner is rarely likely to improve management. However, there may be cases where a patient scheduled for a CT scan has a cardiac arrest just prior to the commencement of the scan. In these circumstances, it may be reasonable to proceed with the scan to confirm the presence of a treatable reversible cause, such as a massive pulmonary 
embolism. In such patients, imaging of acceptable quality can be obtained whilst CPR is delivered by a mechanical device [60].

\section{Extracorporeal CPR}

Extracorporeal CPR (E-CPR) is a cardiac arrest treatment strategy whereby patients are placed on cardiopulmonary bypass. Whilst evidence supporting E-CPR is limited and based on observational studies, a number of regions have established systems where E-CPR may be offered to patients who might narrow inclusion criteria $[61,62]$. Several of these systems use mechanical CPR to facilitate the insertion of the E-CPR intravascular cannulae [63-65].

In Paris, for example, mechanical CPR has been used as a bridge to pre-hospital E-CPR in 156 patients, with an overall ROSC rate of $77.8 \%$ [63]. Similarly, the Australian CHEER study included 26 patients in refractory cardiac arrest that were treated with a care protocol comprising mechanical CPR, therapeutic hypothermia, E-CPR, and pPCI [64]. Fourteen (54\%) survived to hospital discharge, all of whom had full neurological recovery.

The ongoing Prague-based Hyperinvasive Approach in Cardiac Arrest trial (NCT01511666) will provide important new information about the role of mechanical CPR as a bridge to E-CPR [66].

\section{Organ donation}

Uncontrolled donation after circulatory death (uDCD) provides a system whereby organs can be retrieved after sudden cardiac arrest in cases where it has not been possible to obtain a ROSC [67]. This enables the retrieval of organs such as lungs, kidneys, and liver. Whilst this concept poses legal, ethical, and practical challenges, it provides an opportunity to increase the number of viable donor organs $[67,68]$.

The use of mechanical CPR as a bridge to non-heart beating donation has also been described in a number of countries [37,67]. The use of mechanical CPR provides a system to limit warm ischaemic time [68] whilst potentially providing a controlled setting in which consent for donation can be sought. In Spain, a comparable number of organs were transplanted with a similar graft failure rate following implementation of mechanical CPR as part of a uDCD protocol [69]. The study also highlighted the challenge of delivering such a system through its report that three patients, following protocol implementation and initiation of mechanical CPR, obtained ROSC, of which one made a good recovery.

Clinical decisions to refer for advanced lifesaving interventions (e.g. E-CPR) versus organ donation present ethical dilemmas that require careful consideration [70].

\section{Optimising clinical use of mechanical devices Timing of deployment}

In systems where mechanical devices are available, a key challenge for the clinician is the decision as to the time point during the cardiac arrest at which to deploy the mechanical chest compression device. In a meta-regression of out-of-hospital data, Bonnes et al. [71] identified an association between improved outcome and earlier device deployment.

A sub-group analysis in the PARAMEDIC trial identified decreased 30-day survival in patients treated with a mechanical device that presented in a shockable rhythm (odds ratio 0.71, 95\% CI 0.52, 0.98) [19]. One plausible explanation for this is that the study protocol required deployment of the mechanical device prior to defibrillation, leading to delays in defibrillation in the mechanical CPR arm, although this delay was not measured in the trial. In contrast, the LINC study, which adopted a modified mechanical CPR treatment algorithm, found no difference in outcome between treatment groups in patients presenting in a shockable rhythm (e.g. hospital discharge treatment difference $0.6 \%, 95 \%$ CI $-5.6,6.9$ ), despite an increased median time to first shock in the mechanical CPR arm (mechanical 4 min (IQR 2,5) vs manual 3 min (IQR 2, 4), $P<0.001$ ) [72].

Delivery of high-quality manual chest compressions for a prolonged period of time is physically exhausting $[11,73]$. In the context of a prolonged cardiac arrest with limited personnel available, use of a mechanical chest compression device may be a reasonable strategy to avoid the potential harm associated with suboptimal chest compression delivery.

Based on these data, it would seem reasonable to deploy devices early in circumstances where high-quality manual chest compressions cannot be safely delivered. In patients where high-quality CPR is deliverable, delayed deployment would seem prudent in patients in a shockable rhythm.

\section{Deployment}

The key modifiable risk associated with mechanical device use is the pause associated with device deployment. There is a risk that prolonged pauses associated with device deployment during the early part of a cardiac arrest event may offset the subsequent potential benefit of improved CPR.

In clinical practice, published literature reports marked variability in the hands-off time during device deployment, with pauses in excess of 1 minute being reported [74]. In the LINC trial, the median reported chest compression pause associated with device deployment was 36.0 s (IQR 19.5, 45.5) [75]. However, subsequent improvement in flow-fraction following device deployment meant that the median flow-fraction over the first 10 
minutes of the cardiac arrest was higher in the mechanical CPR arm (mechanical 0.84 (IQR 0.78, 0.91) vs manual 0.79 (IQR 0.70, 0.86), $p<0.001$ ). A similar pattern was observed in the CIRC trial [17].

High-quality training that focuses on minimising pauses is an effective strategy to reduce chest compression pauses associated with device deployment $[40,76]$. Levy et al. [76] implemented a system which incorporated a choreographed team approach to device deployment, debriefing, mock resuscitation drills, and adaptations to the deployment process to minimise pauses. The implementation of this system was associated with a significant reduction in the median pause immediately prior to the first mechanical chest compression (21 (IQR 15, 31) vs 7 (IQR 4, 12) s, $p<0.001$ ). Whenever mechanical CPR systems are deployed, a careful system of quality assurance should be initiated to ensure optimal device deployment and avoidance of prolonged interruptions in chest compressions.

\section{Future developments}

The integration of mechanical CPR with other technologies, such as active compression-decompression technology or impedance threshold devices [77], has the potential to impact on the efficacy of currently marketed mechanical chest compression devices. However, a recent study found that the integration of active compression-decompression technology with a LUCAS mechanical chest compression device did not improve end tidal carbon dioxide, compared with use of a LUCAS without the technology [78].

There may be the opportunity in the future for mechanical chest compression devices to titrate chest compression delivery to physiological endpoints, such as end-tidal carbon dioxide or arterial blood pressure [79].

\section{Conclusions}

The provision of high-quality CPR is a key modifiable factor associated with survival in cardiac arrest. Mechanical chest compression devices consistently deliver high-quality chest compressions, but this does not translate into improved patient outcomes when devices are routinely used in OHCA. Further trials are needed to evaluate the routine use of mechanical devices in IHCA.

The use of mechanical devices in specific circumstances (e.g. ambulance/helicopter transport, pPCI) where high-quality chest compressions cannot be safely delivered may be a reasonable strategy. In all situations where mechanical devices are used, clinicians must ensure that the device is deployed with minimal interruption to chest compression delivery.

\section{Abbreviations}

CPR: Cardiopulmonary resuscitation; E-CPR: Extracorporeal cardiopulmonary resuscitation; ED: Emergency department; EMS: Emergency Medical Service;
IHCA: In-hospital cardiac arrest; ILCOR: International Liaison Committee on Resuscitation; OHCA: Out-of-hospital cardiac arrest; pPCl: Primary percutaneous coronary intervention; ROSC: Return of spontaneous circulation; uDCD: Uncontrolled donation after circulatory death

\section{Funding}

KC and JY are supported as NIHR post-doctoral research fellows. GDP is an $\mathrm{NIHR}$ senior investigator.

\section{Authors' contributions}

All authors contributed to the planning and writing of the review. All authors reviewed and approved the final manuscript.

\section{Competing interests}

$\mathrm{KC}$ and GDP have received NIHR funding to evaluate the use of mechanical chest compression devices.

\section{Publisher's Note}

Springer Nature remains neutral with regard to jurisdictional claims in published maps and institutional affiliations.

\section{Author details}

${ }^{1}$ Warwick Medical School, University of Warwick, Coventry, UK. ${ }^{2}$ South Central Ambulance Service NHS Foundation Trust, Bicester, UK. ${ }^{3}$ University Hospitals Birmingham NHS Foundation Trust, Birmingham, UK. ${ }^{4}$ West Midlands Ambulance Service NHS Foundation Trust, Brierly Hill, UK.

Received: 23 November 2017 Accepted: 10 May 2018

Published online: 29 May 2018

\section{References}

1. Perkins GD, Travers AH, Berg RA, Castren M, Considine J, Escalante R, et al. Part 3: Adult basic life support and automated external defibrillation: 2015 International Consensus on Cardiopulmonary Resuscitation and Emergency Cardiovascular Care Science with Treatment Recommendations. Resuscitation. 2015;95:e43-69.

2. Wik L, Kramer-Johansen J, Myklebust H, Sørebø H, Svensson L, Fellows B, Steen PA. Quality of cardiopulmonary resuscitation during out-of-hospital cardiac arrest. JAMA. 2005;293(3):299-304.

3. Abella BS, Alvarado JP, Myklebust H, Edelson DP, Barry A, O'Hearn N, et al. Quality of cardiopulmonary resuscitation during in-hospital cardiac arrest. JAMA. 2005;293(3):305-10.

4. Duchateau FX, Gueye P, Curac S, Tubach F, Broche C, Plaisance P, et al. Effect of the AutoPulse automated band chest compression device on hemodynamics in out-of-hospital cardiac arrest resuscitation. Intensive Care Med. 2010;36(7):1256-60.

5. Axelsson C, Karlsson T, Axelsson AB, Herlitz J. Mechanical active compression-decompression cardiopulmonary resuscitation (ACD-CPR) versus manual CPR according to pressure of end tidal carbon dioxide (PETCO2) during CPR in out-of-hospital cardiac arrest (OHCA). Resuscitation. 2009:80(10):1099-103.

6. Perkins GD, Handley AJ, Koster RW, Castrén M, Smyth MA, Olasveengen T, et al. European Resuscitation Council guidelines for resuscitation 2015: Section 2. Adult basic life support and automated external defibrillation. Resuscitation. 2015;95:81-99.

7. Kleinman ME, Brennan EE, Goldberger ZD, Swor RA, Terry M, Bobrow BJ, et al. Part 5: Adult basic life support and cardiopulmonary resuscitation quality: 2015 American Heart Association Guidelines Update for Cardiopulmonary Resuscitation and Emergency Cardiovascular Care. Circulation. 2015;132(18 suppl 2):S414-35.

8. Wallace SK, Abella BS, Becker LB. Quantifying the effect of cardiopulmonary resuscitation quality on cardiac arrest outcome: a systematic review and meta-analysis. Circ Cardiovasc Qual Outcomes. 2013;6(2):148-56.

9. Talikowska M, Tohira H, Finn J. Cardiopulmonary resuscitation quality and patient survival outcome in cardiac arrest: A systematic review and metaanalysis. Resuscitation. 2015;96:66-77.

10. Jo CH, Cho GC, Ahn JH, Park YS, Lee CH. Rescuer-limited cardiopulmonary resuscitation as an alternative to 2 -min switched CPR in the setting of inhospital cardiac arrest: a randomised cross-over study. Emerg Med J. 2015; 32(7):539-43. 
11. Sugerman NT, Edelson DP, Leary M, Weidman EK, Herzberg DL, Vanden Hoek $T L$, et al. Rescuer fatigue during actual in-hospital cardiopulmonary resuscitation with audiovisual feedback: a prospective multicenter study. Resuscitation. 2009;80(9):981-4.

12. Tomlinson AE, Nysaether J, Kramer-Johansen J, Steen PA, Dorph E. Compression force-depth relationship during out-of-hospital cardiopulmonary resuscitation. Resuscitation. 2007;72(3):364-70.

13. Perkins GD, Kocierz L, Smith SC, McCulloch RA, Davies RP. Compression feedback devices over estimate chest compression depth when performed on a bed. Resuscitation. 2009;80(1):79-82.

14. Nishisaki A, Nysaether J, Sutton R, Maltese M, Niles D, Donoghue A, et al. Effect of mattress deflection on CPR quality assessment for older children and adolescents. Resuscitation. 2009;80(5):540-5.

15. Stiell IG, Brown SP, Nichol G, Cheskes S, Vaillancourt C, Callaway CW, et al. What is the optimal chest compression depth during out-ofhospital cardiac arrest resuscitation of adult patients? Circulation. 2014; 130(22):1962-70.

16. Soar J, Callaway CW, Aibiki M, Böttiger BW, Brooks SC, Deakin CD, et al. Part 4: Advanced life support: 2015 International Consensus on Cardiopulmonary Resuscitation and Emergency Cardiovascular Care Science with Treatment Recommendations. Resuscitation. 2015:95:e71-e120.

17. Wik L, Olsen JA, Persse D, Sterz F, Lozano M, Brouwer MA, et al. Manual vs integrated automatic load-distributing band CPR with equal survival after out of hospital cardiac arrest. The randomized CIRC trial. Resuscitation. 2014; 85(6):741-8.

18. Rubertsson S, Lindgren E, Smekal D, Ostlund O, Silfverstolpe J, Lichtveld RA, et al. Mechanical chest compressions and simultaneous defibrillation vs conventional cardiopulmonary resuscitation in out-of-hospital cardiac arrest: the LINC randomized trial. JAMA. 2014;311(1):53-61.

19. Perkins GD, Lall R, Quinn T, Deakin CD, Cooke MW, Horton J, et al. Mechanical versus manual chest compression for out-of-hospital cardiac arrest (PARAMEDIC): a pragmatic, cluster randomised controlled trial. Lancet. 2015;385(9972):947-55.

20. Hallstrom A, Rea TD, Sayre MR, Christenson J, Anton AR, Mosesso VN Jr, et al. Manual chest compression vs use of an automated chest compression device during resuscitation following out-of-hospital cardiac arrest: a randomized trial. JAMA. 2006:295(22):2620-9.

21. Smekal D, Johansson J, Huzevka T, Rubertsson S. A pilot study of mechanical chest compressions with the LUCAS device in cardiopulmonary resuscitation. Resuscitation. 2011;82(6):702-6.

22. Gates S, Quinn T, Deakin CD, Blair L, Couper K, Perkins GD. Mechanical chest compression for out of hospital cardiac arrest: Systematic review and metaanalysis. Resuscitation. 2015;94:91-7.

23. Dunn G, Maracy M, Dowrick C, Ayuso-Mateos $J$, Dalgard OS, Page H, et al Estimating psychological treatment effects from a randomised controlled trial with both non-compliance and loss to follow-up. Br J Psychiatry. 2003 183:323-31.

24. Ji C, Lall R, Quinn T, Kaye C, Haywood K, Horton J, et al. Post-admission outcomes of participants in the PARAMEDIC trial: a cluster randomised trial of mechanical or manual chest compressions. Resuscitation. 2017:118:82-8.

25. Marti J, Hulme C, Ferreira Z, Nikolova S, Lall R, Kaye C, et al. The costeffectiveness of a mechanical compression device in out-of-hospital cardiac arrest. Resuscitation. 2017;117:1-7.

26. Gates S, Lall R, Quinn T, Deakin CD, Cooke MW, Horton J, et al. Prehospital randomised assessment of a mechanical compression device in out-ofhospital cardiac arrest (PARAMEDIC): a pragmatic, cluster randomised trial and economic evaluation. Health Technol Assess. 2017;21(11):1-176.

27. Morrison LJ, Visentin LM, Kiss A, Theriault R, Eby D, Vermeulen $M$, et al. Validation of a rule for termination of resuscitation in out-of-hospital cardiac arrest. NEJM. 2006;355(5):478-87.

28. Adams BD, Benger J. Should we take patients to hospital in cardiac arrest? BMJ. 2014;349:95659.

29. Hawkes C, Booth S, Ji C, Brace-McDonnell SJ, Whittington A, Mapstone J, et al. Epidemiology and outcomes from out-of-hospital cardiac arrests in England. Resuscitation. 2017;110:133-40.

30. Lyon RM, Crawford A, Crookston C, Short S, Clegg GR. The combined use of mechanical CPR and a carry sheet to maintain quality resuscitation in outof-hospital cardiac arrest patients during extrication and transport. Resuscitation. 2015:93:102-6.

31. Becker LR, Zaloshnja E, Levick N, Li G, Miller TR. Relative risk of injury and death in ambulances and other emergency vehicles. Accid Anal Prev. 2003: 35(6):941-8
32. Kahn CA, Pirrallo RG, Kuhn EM. Characteristics of fatal ambulance crashes in the United States: an 11-year retrospective analysis. Prehosp Emerg Care. 2001;5(3):261-9.

33. Kurz MC, Dante SA, Puckett BJ. Estimating the impact of off-balancing forces upon cardiopulmonary resuscitation during ambulance transport. Resuscitation. 2012;83(9):1085-9.

34. Olasveengen TM, Wik L, Steen PA. Quality of cardiopulmonary resuscitation before and during transport in out-of-hospital cardiac arrest. Resuscitation. 2008;76(2):185-90

35. Cheskes S, Byers A, Zhan C, Verbeek PR, Ko D, Drennan IR, et al. CPR quality during out-of-hospital cardiac arrest transport. Resuscitation. 2017;114:34-9.

36. Roosa JR, Vadeboncoeur TF, Dommer PB, Panchal AR, Venuti M, Smith G, et al. CPR variability during ground ambulance transport of patients in cardiac arrest. Resuscitation. 2013;84(5):592-5.

37. Tazarourte K, Sapir D, Laborne FX, Briole N, Letarnec JY, Atchabahian A, Cornu JF, et al. Refractory cardiac arrest in a rural area: Mechanical chest compression during helicopter transport. Acta Anaesthesiol Scand. 2013; 57(1):71-6.

38. Hayashida K, Tagami T, Fukuda T, Suzuki M, Yonemoto N, Kondo $Y$, et al. Mechanical cardiopulmonary resuscitation and hospital survival among adult patients with nontraumatic out-of-hospital cardiac arrest attending the emergency department: a prospective, multicenter, observational study in Japan (SOS-KANTO [Survey of Survivors after Out-of-Hospital Cardiac Arrest in Kanto Area] 2012 Study). J Am Heart Assoc. 2017;6(11)

39. Ong ME, Fook-Chong S, Annathurai A, Ang SH, Tiah L, Yong KL, et al. Improved neurologically intact survival with the use of an automated, loaddistributing band chest compression device for cardiac arrest presenting to the emergency department. Crit Care. 2012;16(4):R144.

40. Ong ME, Quah JU, Annathurai A, Noor NM, Koh ZX, Tan KBK, et al. Improving the quality of cardiopulmonary resuscitation by training dedicated cardiac arrest teams incorporating a mechanical load-distributing device at the emergency department. Resuscitation. 2013;84(4):508-14.

41. Couper K, Yeung J, Nicholson T, Quinn T, Lall R, Perkins GD. Mechanical chest compression devices at in-hospital cardiac arrest: A systematic review and meta-analysis. Resuscitation. 2016;103:24-31.

42. Lu X, Kang X, Gong D. The clinical efficacy of Thumper modal 1007 cardiopulmonary resuscitation: a prospective randomised control trial. Zhongguo Wei Zhong Bing Ji Jiu Yi Xue. 2010;22(8):496-7.

43. Taylor GJ, Rubin R, Tucker M, Greene HL, Rudikoff MT, Weisfeldt ML. External cardiac compression. A randomized comparison of mechanical and manual techniques. JAMA. 1978;240(7):644.

44. Halperin HR, Tsitlik JE, Gelfand M, Weisfeldt ML, Gruben KG, Levin HR, et al. A preliminary study of cardiopulmonary resuscitation by circumferential compression of the chest with use of a pneumatic vest. N Engl J Med. 1993; 329(11):762-8.

45. Nolan JP, Berg RA, Bernard S, Bobrow BJ, Callaway CW, Cronberg T, et al. Intensive care medicine research agenda on cardiac arrest. Intensive Care Med. 2017:43(9):1282-93.

46. Miller AC, Rosati SF, Suffredini AF, Schrump DS. A systematic review and pooled analysis of CPR-associated cardiovascular and thoracic injuries. Resuscitation. 2014;85(6):724-31.

47. Hoke RS, Chamberlain D. Skeletal chest injuries secondary to cardiopulmonary resuscitation. Resuscitation. 2004:63(3):327-38.

48. Black CJ, Busuttil A, Robertson C. Chest wall injuries following cardiopulmonary resuscitation. Resuscitation. 2004:63(3):339-43.

49. Wind J, Bekkers S, Van Hooren L, Van Heurn L. Extensive injury after use of a mechanical cardiopulmonary resuscitation device. Am J Emerg Med. 2009; 27(8):1017. e1011-2

50. Deras P, Manzanera J, Millet I, Charbit J, Capdevila X. Fatal pancreatic injury due to trauma after successful cardiopulmonary resuscitation with automatic mechanical chest compression. Anesthesiology. 2014;120(4): 1038-41.

51. Shahinian JH, Quitt J, Wiese M, Eckstein F, Reuthebuch O. Unexpected collateral impact after out of hospital resuscitation using LUCAS system. J Cardiothorac Surg. 2017;12(1):81.

52. Smekal D, Johansson J, Huzevka T, Rubertsson S. No difference in autopsy detected injuries in cardiac arrest patients treated with manual chest compressions compared with mechanical compressions with the LUCAS device-a pilot study. Resuscitation. 2009;80(10):1104-7.

53. Smekal D, Lindgren E, Sandler H, Johansson J, Rubertsson S. CPR-related injuries after manual or mechanical chest compressions with the LUCAS 
device: a multicentre study of victims after unsuccessful resuscitation. Resuscitation. 2014;85(12):1708-12.

54. Baumeister R, Held U, Thali MJ, Flach PM, Ross S. Forensic imaging findings by post-mortem computed tomography after manual versus mechanical chest compression. J Forensic Radiol Imaging. 2015;3(3):167-73.

55. Koga Y, Fujita M, Yagi T, Nakahara T, Miyauchi T, Kaneda K, et al. Effects of mechanical chest compression device with a load-distributing band on post-resuscitation injuries identified by post-mortem computed tomography. Resuscitation. 2015;96:226-31.

56. Koster RW, Beenen LF, van der Boom EB, Spijkerboer AM, Tepaske R, van der Wal AC, et al. Safety of mechanical chest compression devices AutoPulse and LUCAS in cardiac arrest: a randomized clinical trial for non-inferiority. Eur Heart J. 2017; https://doi.org/10.1093/eurheartj/ehx318.

57. Wagner H, Hardig BM, Rundgren M, Zughaft D, Harnek J, Gotberg M, et al Mechanical chest compressions in the coronary catheterization laboratory to facilitate coronary intervention and survival in patients requiring prolonged resuscitation efforts. Scand J Trauma Resusc Emerg Med. 2016;24:4.

58. Wagner H, Terkelsen CJ, Friberg H, Harnek J, Kern K, Lassen JF, Olivecrona GK. Cardiac arrest in the catheterisation laboratory: A 5-year experience of using mechanical chest compressions to facilitate $\mathrm{PCl}$ during prolonged resuscitation efforts. Resuscitation. 2010;81(4):383-7.

59. Spiro JR, White S, Quinn N, Gubran CJ, Ludman PF, Townend JN, Doshi SN Automated cardiopulmonary resuscitation using a load-distributing band external cardiac support device for in-hospital cardiac arrest: a single centre experience of AutoPulse-CPR. Int J Cardiol. 2015;180:7-14.

60. Wirth S, Korner M, Treitl M, Linsenmaier U, Leidel BA, Jaschkowitz T, Reiser MF, Kanz KG. Computed tomography during cardiopulmonary resuscitation using automated chest compression devices-an initial study. Eur Radiol. 2009;19(8):1857-66.

61. Kim SJ, Kim HJ, Lee HY, Ahn HS, Lee SW. Comparing extracorporeal cardiopulmonary resuscitation with conventional cardiopulmonary resuscitation: A meta-analysis. Resuscitation. 2016;103(Supplement C): 106-16.

62. Ortega-Deballon I, Hornby L, Shemie SD, Bhanji F, Guadagno E. Extracorporeal resuscitation for refractory out-of-hospital cardiac arrest in adults: a systematic review of international practices and outcomes. Resuscitation. 2016;101:12-20.

63. Lamhaut L, Hutin A, Puymirat E, Jouan J, Raphalen JH, Jouffroy R, et al. Prehospital extracorporeal cardio pulmonary resuscitation (ECPR) strategy for treatment of refractory out hospital cardiac arrest: an observational study and propensity analysis. Resuscitation. 2017;117:109-17.

64. Stub D, Bernard S, Pellegrino V, Smith K, Walker T, Sheldrake J, et al. Refractory cardiac arrest treated with mechanical CPR, hypothermia, ECMO and early reperfusion (the CHEER trial). Resuscitation. 2015;86:88-94.

65. Yannopoulos D, Bartos JA, Raveendran G, Conterato M, Frascone RJ, Trembley A, et al. Coronary artery disease in patients with out-of-hospital refractory ventricular fibrillation cardiac arrest. J Am Coll Cardiol. 2017;70(9): 1109-17.

66. Belohlavek J, Kucera K, Jarkovsky J, Franek O, Pokorna M, Danda J, et al. Hyperinvasive approach to out-of hospital cardiac arrest using mechanical chest compression device, prehospital intraarrest cooling, extracorporeal life support and early invasive assessment compared to standard of care. A randomized parallel groups comparative study proposal. "Prague OHCA study". J Transl Med. 2012;10:163.

67. Ortega-Deballon I, Hornby L, Shemie SD. Protocols for uncontrolled donation after circulatory death: a systematic review of international guidelines, practices and transplant outcomes. Crit Care. 2015;19:268.

68. Manara AR, Murphy PG, O'Callaghan G. Donation after circulatory death. Br J Anaesth. 2012;108(suppl_1):i108-21.

69. Mateos-Rodriguez A, Pardillos-Ferrer L, Navalpotro-Pascual JM, Barba-Alonso C, Martin-Maldonado ME, Andres-Belmonte A. Kidney transplant function using organs from non-heart-beating donors maintained by mechanical chest compressions. Resuscitation. 2010;81(7):904-7.

70. Dalle Ave AL, Shaw DM, Gardiner D. Extracorporeal membrane oxygenation (ECMO) assisted cardiopulmonary resuscitation or uncontrolled donation after the circulatory determination of death following out-of-hospital refractory cardiac arrest-an ethical analysis of an unresolved clinical dilemma. Resuscitation. 2016;108:87-94.

71. Bonnes JL, Brouwer MA, Navarese EP, Verhaert DVM, Verheugt FWA, Smeets $J L R M$, de Boer M-J. Manual cardiopulmonary resuscitation versus CPR including a mechanical chest compression device in out-of-hospital cardiac arrest: a comprehensive meta-analysis from randomized and observational studies. Ann Emerg Med. 2016;67(3):349-60.

72. Hardig BM, Lindgren E, Östlund O, Herlitz J, Karlsten R, Rubertsson S. Outcome among VFNT patients in the LINC (LUCAS IN cardiac arrest) trial-a randomised, controlled trial. Resuscitation. 2017;115:155-62.

73. Shin J, Hwang SY, Lee HJ, Park CJ, Kim YJ, Son YJ, et al. Comparison of CPR quality and rescuer fatigue between standard 30:2 CPR and chest compressiononly CPR: a randomized crossover manikin trial. Scand J Trauma Resusc Emerg Med. 2014;22:59

74. Yost D, Phillips RH, Gonzales L, Lick CJ, Satterlee P, Levy M, et al. Assessment of CPR interruptions from transthoracic impedance during use of the LUCAS mechanical chest compression system. Resuscitation. 2012;83(8):961-5.

75. Esibov A, Banville I, Chapman FW, Boomars R, Box M, Rubertsson S. Mechanical chest compressions improved aspects of CPR in the LINC trial. Resuscitation. 2015;91:116-21.

76. Levy M, Yost D, Walker RG, Scheunemann E, Mendive SR. A quality improvement initiative to optimize use of a mechanical chest compression device within a high-performance CPR approach to out-of-hospital cardiac arrest resuscitation. Resuscitation. 2015;92:32-7.

77. Aufderheide TP, Frascone RJ, Wayne MA, Mahoney BD, Swor RA, Domeier RM, et al. Standard cardiopulmonary resuscitation versus active compression-decompression cardiopulmonary resuscitation with augmentation of negative intrathoracic pressure for out-of-hospital cardiac arrest: a randomised trial. Lancet. 2011;377(9762):301-11.

78. Berve P-O, Skålhegg T, Madsen Hardig B, Carlson J, Kramer-Johansen J, Wik L. Mechanical active compression-decompression with LUCAS2AD provide equivocal $\mathrm{pEtCO}$ values to the standard LUCAS2 during out-of-hospital cardiopulmonary resuscitation. Circulation. 2017;136(Suppl 1):A18119.

79. Sundermann ML, Salcido DD, Koller AC, Menegazzi JJ. Feasibility of biosignal-guided chest compression during cardiopulmonary resuscitation: a proof of concept. Acad Emerg Med. 2016;23(1):93-7. 